\title{
Orchiepididymitis in a 14-year-old boy with concurrent SARS-CoV-2 infection
}

\author{
M Wronowski, ${ }^{1} \mathrm{PhD} ; \mathbf{W}$ Woźniak, ${ }^{1} \mathrm{PhD} ; \mathbf{M}$ Wanke-Rytt, ${ }^{1} \mathrm{PhD} ; \mathbf{K}$ Jażdzewski, ${ }^{2} \mathrm{PhD}$; E Kuchar, ${ }^{1} \mathrm{PhD} ; \mathbf{M}$ Kloc, ${ }^{3,4,5} \mathrm{PhD} ;$ \\ J Z Kubiak, ${ }^{6,7,8} \mathrm{PhD}$ \\ ${ }^{1}$ Department of Pediatrics with Clinical Assessment Unit, Medical University of Warsaw, Poland \\ ${ }^{2}$ Department ofGenomic Medicine, Medical University of Warsaw, Poland \\ ${ }^{3}$ The Houston Methodist Research Institute, Texas, USA \\ ${ }^{4}$ The Houston Methodist Hospital, Department of Surgery, Texas, USA \\ ${ }^{5}$ MD Anderson Cancer Center, Department of Genetics, University of Texas, Houston, USA \\ ${ }^{6}$ Laboratory of Regenerative Medicine and Cell Biology, Military Institute of Hygiene and Epidemiology, Warsaw, Poland \\ ${ }^{7}$ UnivRennes, Institute of Genetics and Development of Rennes, Faculty of Medicine, Rennes, France \\ ${ }^{8}$ Department of Experimental Embryology, Institute of Genetics and Animal Biotechnology of the Polish Academy of Sciences, Jastrzebiec, Poland
}

Corresponding author: E Kuchar (Ernest.kuchar@wum.edu.pl)

The symptoms of SARS-CoV-2 infection and COVID-19 provoked by this virus are poorly described in children. Here we analyse a case of orchiepididymitis associated with COVID-19 in a 14-year-old boy. We discuss the possibility of SARS-CoV-2-associated testicular inflammation. This report strengthens the necessity for more in-depth study of the clinical presentation of paediatric COVID-19 and the potential association with non-respiratory symptoms.

S Afr J Child Health 2021;15(4):227-228. https://doi.org/10.7196/SAJCH.2021.v15i4.1869

Severe acute respiratory syndrome coronavirus 2 (SARS-CoV-2) provoking coronavirus disease 2019 (COVID-19) was first described in Wuhan, China, in December 2019. Within months, COVID-19 spread rapidly worldwide, becoming the first pandemic of the 21 st century. COVID-19 produces mainly mild symptoms in most infected children. However, it can cause a plethora of inflammatory complications including multisystem inflammatory syndrome in children (MIS-C). Since COVID-19 is an emerging infectious disease, there are limited data about the effects of this infection on patients, especially in the paediatric population. We describe here a case of the co-occurrence of orchiepididymitis and SARS-CoV-2 infection in a 14-year-old adolescent. As a similar case has already been reported by Gagliardi et al., ${ }^{[1]}$ our description is a potential next argument for the possible link between orchiepididymitis and COVID-19.

\section{Case report}

On 27 March 2020, a 14-year-old boy had a fever $\left(39.4^{\circ} \mathrm{C}\right)$, complained of dysuria, vesical tenesmus and frequent urination. Owing to the COVID-19 pandemic, the patient telephoned his general practitioner, who diagnosed cystitis and prescribed cefuroxime axetil. Resolution of dysuria was observed one day after treatment. However, the patient developed a mild dry cough when moving.

Because of anorectal pain, on 29 March the patient presented at Jan Bogdanowicz Children's Hospital in Warsaw, where swelling of the scrotum was observed; the testicles were enlarged and painful on palpation, predominantly on the right side. The child was admitted to this hospital's paediatric emergency department, where an ultrasonogram (USG) revealed testicular torsion. Laboratory tests showed neutrophilic leukocytosis (white blood cells
$20700 \mathrm{~K} / \mu \mathrm{L}$, neutrophils $17400 \mathrm{~K} / \mu \mathrm{L}$ ) with elevated C-reactive protein $(85.2 \mathrm{mg} / \mathrm{L})$. Urinalysis showed leukocyturia and leukocyte aggregates. On admission to the hospital, a nasopharyngeal swab for SARS-CoV-2 reverse transcription polymerase chain reaction (RT-PCR) test was taken.

A surgical revision of the scrotum was performed, showing no testicular torsion but inflammation of the epididymis. The day after surgery, a positive result for the SARS-CoV-2 PCR test was received. The social history revealed that the patient's father had been in contact with international lorry drivers. It was of interest because, by the end of March 2020, the COVID-19 epidemic was not yet widespread in Poland, and foreign contacts could be the source of SARS-CoV-2 infection. The father of the patient had not been tested for SARS-CoV-2. The patient and his father were referred to the Medical University of Warsaw Pediatric Teaching Hospital COVID-19 ward on 30 March because of the positive PCR test for SARS-CoV-2 and mild respiratory symptoms.

Here, we performed a USG follow-up because of persistent inguinal pain. The imaging detected thickening of the tunica albuginea, vasitis, and epididymitis features. We switched antibiotic therapy to one-dose ceftriaxone and oral levofloxacin. This treatment resolved scrotal oedema and testicular pain within three days of hospitalisation. We took a urine sample for the SARS CoV-2 RT-PCR test on 2 April, which was negative.

A second swab was taken nine days after the first swab according to the criteria for releasing COVID-19 patients from isolation published by the World Health Organization in January 2020. This PCR test was negative. The patient was discharged home with the recommendation of 14-day family quarantine under the county sanitary agency's surveillance. 


\section{Discussion}

The clinical presentations of SARS-CoV-2 infection in children are highly diverse. Respiratory symptoms are among the most common, with a cough occurring in $40 \%$ of children. Our patient presented with only mild respiratory symptoms. However, other systems may be affected, among them the urogenital being one of the rarest.

High levels of the receptor for angiotensin-converting enzyme 2 (ACE2) were found in the testicular spermatogonia and Leydig and Sertoli cells. ACE2 receptors allow the virus to infect the cells and, possibly, cause damage, even in asymptomatic cases. In individuals with respiratory symptoms, SARS-CoV-2 RNA can be detected in semen during the acute phase of COVID-19 and later. ${ }^{[2]}$

Epididymal cells may also be the target for the virus. Infected individuals have a higher concentration of human epididymal protein 4 (HE4) than healthy individuals. ${ }^{[3]}$

Despite the ongoing COVID-19 pandemic, only a few cases of symptomatic testicular involvement in adults and children have been described. Testicular pain and fever were the dominant symptoms. ${ }^{[4,5]}$ For this reason, there is a debate whether COVID-19 may be involved in the aetiology of testicular complications. ${ }^{[1]}$ Our case report brings new light to the possibility.

The history of our patient resembles the case reported by Gagliardi et al. ${ }^{[1]}$ The boy was treated with an antibiotic first, and when scrotal swelling progressed, and high fever and pain persisted, he was suspected of having an acute scrotum. In addition, our patient underwent an unnecessary surgical revision of the scrotum. It must be noted that our patient is only the second case of concomitant orchiepididymitis and SARS CoV-2 infection reported at the time of writing this report, after one year of the COVID-19 pandemic. The previous case also concerned a child; no case involving an adult has been described so far. It seems, therefore, that the orchiepididymitis related to SARS-CoV-2 infection may be child-specific. Moreover, COVID-19 produces mild or no symptoms in children and only the severest orchiepididymitis cases present to hospitals, where routine PCR tests for SARS-CoV-2 are done, as in the case described here.
This may explain why the relationship between orchiepididymitis and SARS-CoV-2 infection is still ignored.

\section{Conclusions}

In the COVID-19 era, SARS-CoV-2 infection should be included in the differential diagnosis of orchiepididymitis, and the use of personal protective equipment considered for the medical staff. Moreover, in the co-occurrence of orchiepididymitis and SARSCoV-2 infection, the use of antibiotics for treatment may not be justified.

Acknowledgements. We thank the patient and his family for consent to publication.

Author contributions. Equal contributions.

Funding. While writing this article, JZK was supported by the grant Kościuszko \#508/2017/DA from the Polish Ministry of National Defence. Conflicts of interest. None.

1. Gagliardi L, Bertacca C, Centenari C, et al. Orchiepididymitis in a boy with COVID-19. Pediatr Infect Dis J 2020;39:e200-e202. https://doi.org/10.1097/ INF.0000000000002769

2. Li D, Jin $\mathrm{M}$, Bao $\mathrm{P}$, et al. Clinical characteristics and results of semen tests among men with coronavirus disease 2019. JAMA Netw Open 2020;3(5):e208292. https://doi.org/10.1001/jamanetworkopen.2020.8292.

3. Li T, Wang L, Wang H, et al. Characteristics of laboratory indexes in COVID-19 patients with non-severe symptoms in Hefei City, China: Diagnostic value in organ injuries. Eur J Clin Microbiol Infect Dis 2020;39(12):2447-2455. https://doi.org/10.1007/s10096-020-03967-9.

4. La Marca A, Busani S, Donno V, et al. Testicular pain as an unusual presentation of COVID-19: A brief review of SARS-CoV-2 and the testis. Reprod Biomed Online 2020; 41(5):903-906. https://doi.org/10.1016/j. rbmo.2020.07.017

5. Kim J, Thomsen T, Sell N, Goldsmith AJ. Abdominal and testicular pain: An atypical presentation of COVID-19. Am J Emerg Med 2020;38(7):1542.e11542.e3. https://doi.org/10.1016/j.ajem.2020.03.052. 